\title{
Multimillijoule Sub-Optical-Cycle Parametric Waveform Synthesis for Attosecond Science
}

\author{
Giovanni Cirmi, ${ }^{1,3}$ Shaobo Fang, ${ }^{1,3}$ Shih-Hsuan Chia, ${ }^{1,3}$ Oliver D. Mücke, ${ }^{1,3}$ \\ Franz X. Kärtner, ${ }^{1-4}$ Cristian Manzoni, ${ }^{5}$ Paolo Farinello, ${ }^{5}$ and Giulio Cerullo ${ }^{5}$ \\ ${ }^{1}$ Center for Free-Electron Laser Science, Deutsches Elektronen-Synchrotron DESY, Notkestr. 85, 22607 Hamburg, Germany \\ ${ }^{2}$ Physics Department, University of Hamburg, Luruper Chaussee 149, 22761 Hamburg, Germany \\ ${ }^{3}$ The Hamburg Center for Ultrafast Imaging, Luruper Chaussee 149, 22761 Hamburg, Germany \\ ${ }^{4}$ Department of Electrical Engineering and Computer Science and Research Laboratory of Electronics, \\ Massachusetts Institute of Technology, Cambridge, Massachusetts 02139, USA \\ ${ }^{5}$ IFN-CNR, Dipartimento di Fisica, Politecnico di Milano, Piazza Leonardo da Vinci 32, I-20133 Milan, Italy
}

\begin{abstract}
We present a phase-stable, multi-mJ 3-channel parametric synthesizer generating 2-octave-wide waveforms (0.52-2.4 $\mu \mathrm{m})$. After two amplification stages, the combined $125-\mu \mathrm{J}$ output supports $1.9-\mathrm{fs}$ waveforms. The energy is scaled to $2 \mathrm{~mJ}$ after three amplification stages.
\end{abstract}

\section{INTRODUCTION}

The coherent synthesis of custom-tailored, intense, few-cycle or even sub-cycle optical waveforms is currently one of the most intriguing and promising frontiers of attosecond science and strong-field physics, opening up unprecedented prospects, e.g., for precision control of strong-field interactions in atoms, molecules and solids, for the generation of intense isolated attosecond pulses, and for attosecond pump-probe spectroscopy employing ultrashort pulses in the VIS/IR and XUV/soft-X-ray regions. The key ingredients of coherent pulse synthesis are (i) the generation of an ultrabroad spectrum, (ii) extremely precise dispersion control over the whole bandwidth, (iii) sub-cycle timing synchronization as well as tight stabilization of the relative phases between the different pulses, and (iv) carrier-envelope phase (CEP) stability required for generating reproducible electric-field transients.

Recently, coherent pulse synthesis based on supercontinuum generation in a neon-filled hollow-core fiber compressor allowed the generation of sub-cycle $\sim 300-\mu \mathrm{J}$ optical pulses $(\sim 250 \mu \mathrm{J}$ energy in the NIR channel, $\sim 35$ $\mu \mathrm{J}$ in the VIS, and $\sim 15 \mu \mathrm{J}$ in the VIS-UV) [1]. However, the energy throughput of such hollow-core fiber compressors is limited by ionization losses in the gas medium, thus preventing further energy upscaling to the (multi-)mJ level, which is required for many interesting applications in attosecond science. In contrast, parametric synthesizers [2-6] do not face an energy-scaling bottleneck, and spectral extension into the mid-IR region is particularly appealing for the realization of bright coherent tabletop high-harmonic sources in the waterwindow and keV X-ray region [7].

In our earlier works, we already demonstrated coherent pulse synthesis between ultrabroadband $870-\mathrm{nm}$ and $2.15-\mu \mathrm{m}$ pulses based on optical parametric chirped-pulse amplification (OPCPA) resulting in a $15-\mu \mathrm{J}$ sub-cycle waveform [2] and also between two optical parametric amplifiers (OPAs) with 1-2 $\mu \mathrm{J}$ energy each [3]. Here, we present our ongoing development of a novel multi-mJ 3channel parametric synthesizer (shown in Fig. 1) for generating a 2-octave-wide spectrum with $\sim 1.9$-fs transform-limited (TL) pulse duration.

\section{RESULtS}

Starting point is a cryogenically cooled Ti:sapphire chirped-pulse amplifier (150 fs, $18 \mathrm{~mJ}, 0.8 \mu \mathrm{m}, 1 \mathrm{kHz})$. We generate a CEP-stable continuum $(0.5-2.5 \mu \mathrm{m})$ [5] by white-light generation in a YAG crystal pumped by the second harmonic $(1.06 \mu \mathrm{m})$ of the CEP-stable idler of a NIR OPA. The continuum is split with custom-designed dichroic beam splitters (which will also be used for the final beam recombination) and seeds three OPA channels, a VIS non-collinear OPA (NOPA), a NIR and an IR degenerate OPA (DOPA) channel, pumped by the pulses at $0.8 \mu \mathrm{m}$ (IR DOPA) and its second harmonic at $0.4 \mu \mathrm{m}$

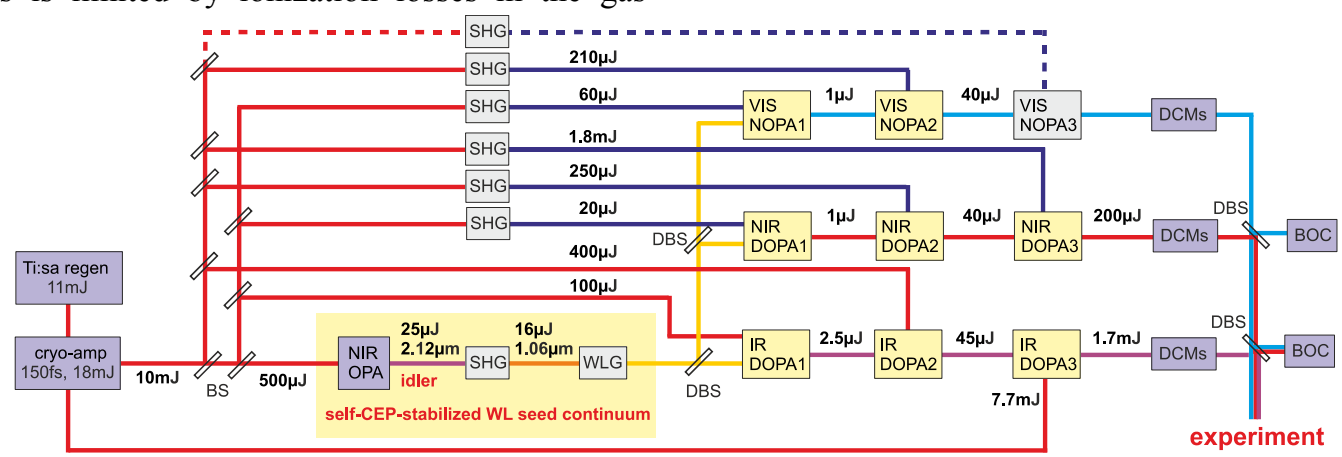

Fig. 1. Scheme of the 2-octave-wide multi-mJ parametric synthesizer. BS, beam splitter; SHG, second-harmonic generation; WLG, white-light generation; DBS, dichroic beam splitter; NOPA, non-collinear OPA; DOPA, degenerate OPA; DCMs, double-chirped mirrors; BOC, balanced optical cross-correlator. 1.7- $\mathrm{mJ} 0.4-\mu \mathrm{m}$ pump pulses can be used for energy scaling of the VIS NOPA3 and NIR DOPA3. For example, using all 1.7 $\mathrm{mJ}$ of the $0.4-\mu \mathrm{m}$ pulses, we obtain $200 \mu \mathrm{J}$ output from the NIR DOPA3, or the pump energy can be split between VIS NOPA3 and NIR DOPA3. 
(VIS NOPA, NIR DOPA). All OPAs employ type-I BBO crystals (the phase-matching angle $\theta$ is $31^{\circ}, 29^{\circ}$, and $20^{\circ}$ for the VIS NOPA, NIR DOPA and IR DOPA, respectively). After parametric amplification, the three OPA channel outputs can individually be recompressed using custom-tailored double-chirped mirrors (DCMs). Finally, in order to synthesize a coherent ultrashort waveform from these OPAs, the relative timing of the pulses will be tightly locked using feedback loops with balanced optical cross-correlators (BOCs), that can achieve sub-cycle synchronization with $<30$-as RMS timing jitter [2,3].
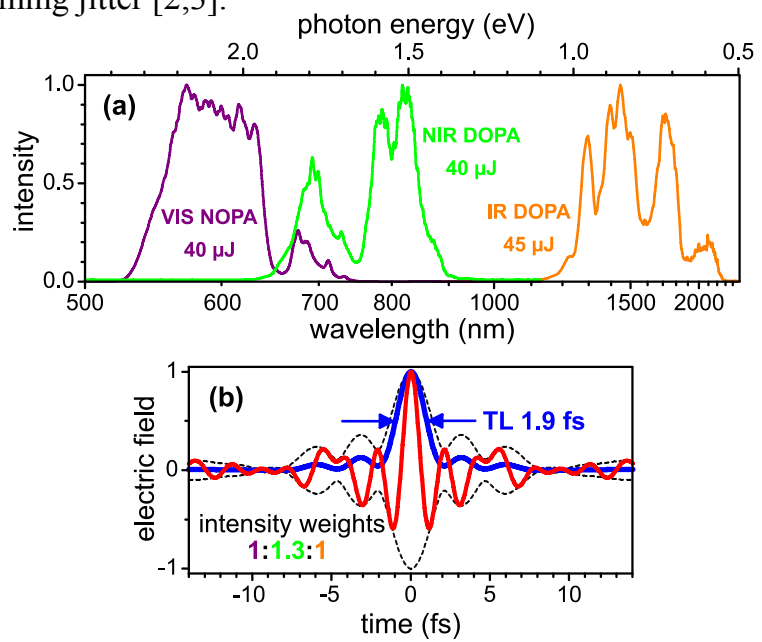

Fig. 2. (a) Output spectra and energies from the second OPA stages. (b) The transform-limited (TL) pulse synthesized from the weighted spectra in (a) and assuming all CEPs to be 0 corresponds to a 1.9-fs FWHM pulse duration. The red curve is the electric field $E(t)$, the dashed curves indicate the field envelope, the intensity profile $I(t)$ is the blue trace.

Figure 2(a) shows the measured output spectra from the second amplification stages operating in parallel. The VIS NOPA and NIR DOPA continuously cover the region from $520 \mathrm{~nm}$ to $960 \mathrm{~nm}$, the IR DOPA spectrum extends from $1130 \mathrm{~nm}$ to $2290 \mathrm{~nm}$. The TL pulse duration from the synthesis of these three spectra (assuming 1:1.3:1 intensity weighting and all CEPs to be 0 ) is $1.9 \mathrm{fs}$ FWHM, corresponding to 0.7 optical cycles at $785 \mathrm{~nm}$ center wavelength, as shown in Fig. 2(b).

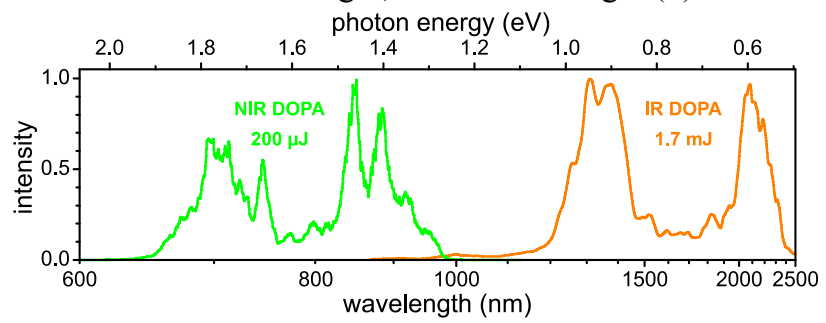

Fig. 3. Output spectra from the third stages of the NIR and IR DOPAs. These spectra, which are not contaminated by superfluorescence backgrounds, both support 5.2-fs pulses corresponding to 2.1/1.1 optical cycles at $750 \mathrm{~nm} / 1390 \mathrm{~nm}$ center wavelengths, respectively. Note that the bandwidth of the second stages were slightly increased compared to the spectra shown in Fig. 2, to optimize the seeding of the third stages.

Afterwards, the energy can be scaled to the multi-mJ level in the third amplification stages (see Fig. 3). First, the energy of the IR DOPA is increased to $1.7 \mathrm{~mJ}$ in a third IR DOPA stage (also see Fig. 4) employing a 4- mm-thick BBO crystal pumped by $7.7 \mathrm{~mJ}$ pulses, i.e., we achieve a pump-signal conversion efficiency of $22 \%$. Second, depending on the requirements of the attosecond experiment, that we want to perform in the future, we can split the remaining $1.7-\mathrm{mJ} 0.4-\mu \mathrm{m}$ pump pulses for scaling the energy in VIS NOPA3 and NIR DOPA3. For example, using all $1.7 \mathrm{~mJ}$ of $0.4-\mu \mathrm{m}$ pump pulses and a 2mm-thick BBO, we obtain $200 \mu \mathrm{J}$ output from the NIR DOPA3 (see Fig. 3).

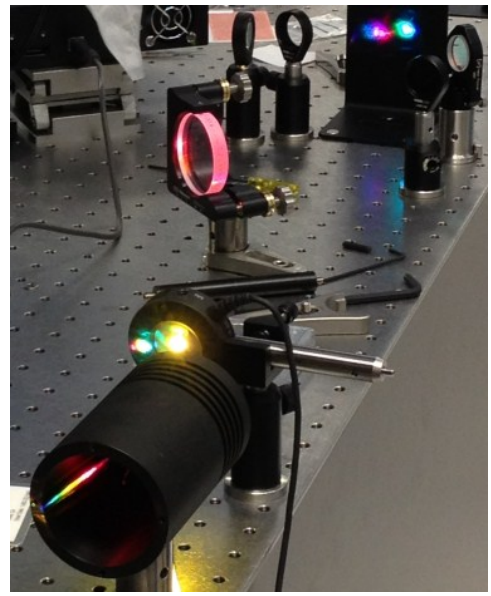

Fig. 4. IR DOPA3 output. Due to the high energy in this stage, various visible components are generated by parasitic nonlinearities in the $\mathrm{BBO}$ crystal. An energy-meter was used to optimize the amplified signal energy, all light components from the idler are blocked inside the beam dump in the front. Note that the $1.7 \mathrm{~mJ}$ signal energy was measured behind a long-pass filter transmitting only wavelengths $>1150 \mathrm{~nm}$.

The enabling technology for the final pulse compression to a 1.9 -fs duration and successful beam recombining (avoiding $B$-integral issues) are state-of-theart DCM pairs with ultralow residual ripple in the resulting total group-delay dispersion over the full bandwidth, which have been fabricated and characterized. Temporal characterization of the synthesized two-octavespanning optical waveforms will be performed by twodimensional spectral shearing interferometry (2DSI) $[2,3]$.

\section{CONCLUSIONS AND OUTLOOK}

We foresee that our multi-mJ 2-octave-wide 3-channel synthesizer will become a versatile tool for controlling strong-field interactions in atoms, molecules and solids and for attosecond pump-probe spectroscopy employing ultrashort pulses in the VIS/IR and XUV/soft-X-ray regions.

\section{REFERENCES}

[1] A. Wirth et al., Science 334, 195 (2011); M. T. Hassan et al., Rev. Sci. Instrum. 83, 111301 (2012).

[2] S.-W. Huang et al., Nature Photonics 5, 475 (2011); S.-W. Huang et al., J. Phys. B 45, 074009 (2012).

[3] C. Manzoni et al., Opt. Lett. 37, 1880 (2012).

[4] E. J. Takahashi et al., Phys. Rev. Lett. 104, 233901 (2010).

[5] C. Vozzi et al., Phys. Rev. A 79, 033842 (2009).

[6] G. Cerullo et al., Laser \& Photonics Rev. 5, 323 (2011).

E. J. Takahashi et al., Phys. Rev. Lett. 101, 253901 (2008); T. Popmintchev et al., Science 336, 1287 (2012). 